\title{
Hysteresis and the Limits of Homeostasis: From Daisyworld to Phototaxis
}

\author{
James Dyke and Inman Harvey \\ Evolutionary and Adaptive Systems Group, \\ Centre for Computational Neuroscience and Robotics \\ Informatics, School of Science \& Technology \\ University of Sussex, BN1 9QH, UK \\ j.g.dyke@sussex.ac.uk, inmanh@sussex.ac.uk
}

\begin{abstract}
All biological organisms must be able to regulate certain essential internal variables, e.g. core body temperature in mammals, in order to survive. Almost by definition, those that cannot are dead. Changes that result in a mammal being able to tolerate a wider range of core body temperatures make that organism more robust to external perturbations. In this paper we show that when internal variables are regulated via 'rein control' mechanisms, decreasing the range of tolerable values increases the area of observed hysteresis but does not decrease the limits of regulation. We present circumstances where increasing the range of tolerable values actually decreases robustness to external perturbation.
\end{abstract}

\section{Introduction}

In a biological context, the term homeostasis is applied to the inherent tendency in an organism toward maintenance of physiological stability. For example, mammals must maintain core body temperature to within a certain range if they are to survive. Mechanisms to maintain a minimum core body temperature could be shivering and reduction of blood circulation to the extremities. If core body temperature increases to the upper limits of this viability range, then sweating and dilation of capillaries will lower core body temperature. Following Ashby [1] we define such internal variables as essential variables. Furthermore we define the tolerance - the range of values that the essential variable must be maintained within - as the essential range. For example core body temperature in Homo sapiens must be maintained within the essential range of approximately 35-41 degrees Celsius.

In this paper we will argue that for systems that are regulated via 'rein control' (as discussed below), decreasing the essential range may not decrease the range over which homeostasis is performed. We will demonstrate that increasing the essential range may actually decrease robustness to external perturbation. Clynes [2] postulated that many physiological homeostatic processes operate on the basis of opposing control reins that each pull in a single direction in response to certain variables; in order to regulate for both upper and lower limits, two reins, two separate mechanisms 
are required. For a physiological application of rein control see Saunders et al [4] who employ Clynes' rein control analysis to understand the mechanisms responsible for the regulation of blood sugar levels in humans.

We will show that altering the essential range changes the area of hysteresis. Hysteresis will be observed in a system when the output or behaviour is bistable as an input parameter that represents some property of the system is changed through some range of values; when the input is increased through that range, the output is one function of the input, yet if the input decreases, the output is a different function of the input, thus tracing out a 'hysteresis loop'.

In order to explore these issues, analysis will be carried out on the behaviour of a simple two-box 'cable car' model that performs phototaxis. This model is based on the radically simplified Daisyworld model, as detailed by Harvey in [3]. The original Watson \& Lovelock Daisyworld model [5] was intended to demonstrate the homeostatic properties of a planet that is covered with varying proportions of black and white daisies. Watson \& Lovelock employ the Stefan-Boltzmann law to determine the temperatures of the daisies and bare earth. While such an approach involves a non-linear change in temperature with respect to absorbed energy, the relationship between the albedo and the temperature of a body is straightforward; given a fixed amount of short-wave radiation, the lower the albedo, the darker the body, the less radiation is reflected and so the higher the temperature. When the star that heats the planet is dim, the planet is cool. Black daisies, having a lower albedo than either white daisies or the bare earth absorb more of the radiated short-wave energy from the star and so will be warmer than either white daisies or bare earth. If the brightness or luminosity of the star steadily increases, then black daisies will begin to grow. As the proportional coverage of black daisies increases, the net albedo of the planet decreases. This raises the temperature and so increases the rate of daisy growth. The result of this positive feedback is a population explosion of black daisies and a sharp increase in the planetary and daisy temperatures. If luminosity continues to increase, the planet eventually becomes too warm for black daisies to be able to grow. Only white daisies are cool enough to survive as they reflect a greater proportion of the incoming solar radiation. In this way, the black and white daisies regulate the planetary temperature, keeping it within the essential range over a wider range of luminosities than would be the case with a bare lifeless planet.

Rather than formulating an abstract model of a homeostatic system, we instead follow the precedent of Daisyworld and present the cable car model in the form of a 'parable'. To that end, the simplification process begun in [3] is taken further. The cut-down 'toy' physics is reduced to simple linear responses to a light source position whilst the relationship between temperature and albedo is dispensed with. These further simplifications will aid investigation into the relationship between homeostasis, hysteresis, essential range values, and in particular demonstrate that increasing the essential range of the model decreases the area of observed hysteresis but does not increase the limits of self-regulation. Furthermore there are circumstances where increasing the range of tolerable values actually decreases the limits of self-regulation. 


\subsection{Organisation of Paper}

The cable car model will be introduced and compared to Daisyworld in the following section. Both models are composed of two control reins, loosely coupled via their interaction with a shared external driving force. Results from the cable car model will be presented in Section 3. Section 4 will analyse the results. Section 5 concludes the paper.

\section{The Cable Car Model}

The model is based on the cable cars found in San Francisco. Unlike the systems used in the Alps and other mountainous regions, the San Francisco system consists of cables that are located under the road surface and connect to tram like cars. In our model a photovoltaic cell - a 'solar panel' - is located on the roof and supplies current to an electric motor which instead of being located in a winding house, is carried within the cable car itself. As the motor turns, it pulls in a cable that moves the car up the side of a valley. The output of the solar panel, and therefore the force that the motor produces, changes linearly with varying inclination from a moveable light source. When the light source is directly overhead, maximum output is produced and so maximum motor output is achieved. Deviations left or right by either the cable car or light source result in decreasing energy production. The range of light source locations that produce current in the solar panel we call the activation range and is analogous to the essential range of viable daisy temperatures in Daisyworld. It is assumed that the light source is so far away (e.g. the sun) that the energy input depends solely on relative angle to vertical, and any distance change is irrelevant. This does not make any substantive difference to the behaviour of the model but does allow easier analysis.

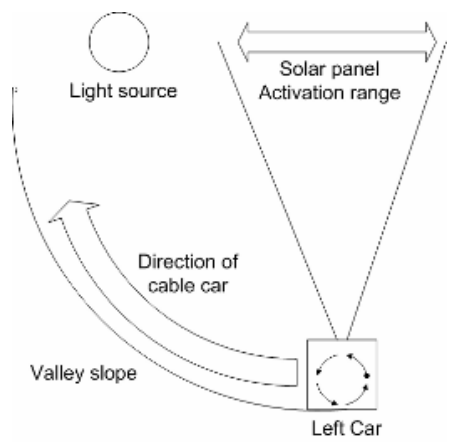

Fig. 1. As the light source enters the activation range of the solar panel, the motor rotates anticlockwise, pulling on the cable which moves the car to the left and so up the valley slope. The gradient of the slope can be understood to increase non-linearly, e.g. the valley has a ' $U$ ' shape and so the car experiences an increasing 'resisting' force due to gravity as it moves further from its starting position.

As the light moves into the left-hand side of the activation range, the solar panel will begin to produce current. The motor will turn anti-clockwise pulling in the cable 
and so move the car to the left. This will bring the light nearer the centre of the solar panel's activation range and further increase the motor output, and move the car further to the left, higher up the valley slope. The cable car has a dimensionless mass of 1 unit. As the car moves further from its starting position, the gradient of the slope increases and so the 'resisting' force due to gravity pulling the car back to the bottom of the valley, $\gamma$, increases:

$$
\gamma=\eta \frac{X}{l} .
$$

Where $X$ is the position of the car in dimensionless x-units, $l$ is the length of the slope and $\eta$ measures the rate of increase of the resisting force as the car travels higher up the slope. For the simulations presented in this paper $\eta$ was set to 1 and so $\gamma$ increases linearly from 0 when the car is at the bottom, to 1 when the car is at the top of the valley.

$\alpha$ is the force produced by the motor. This was set to vary linearly from 0 to a maximum of 1 in response to the output of the solar panel:

$$
\alpha=\operatorname{Max}\left[1-2\left(\left|X_{\text {light }}-X\right|\right) / \varphi, 0\right]
$$

where $X_{\text {light }}$ is the location of the light source and $\varphi$ is the activation range of the solar panel. This produces a 'witches hat' shaped activation function that can be understood as a piece-wise linear version of the original Daisyworld parabolic growth function.

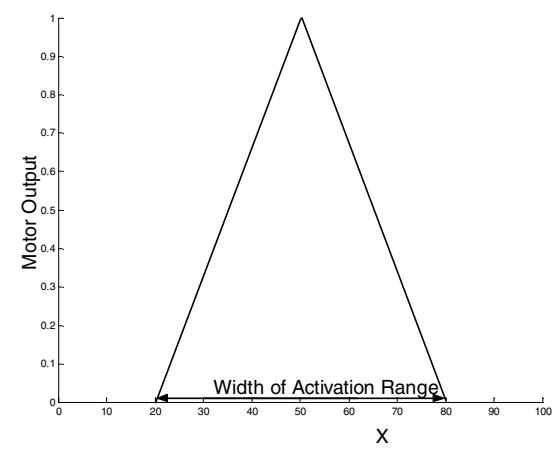

Fig. 2. The output of the solar panel is maximized and so motor output is greatest when the light is directly overhead. As the light moves away from this point, motor output decreases linearly.

The model is completed with the introduction of another cable car that moves up the opposite side of the valley.

The energy provided by the solar panel turns the motor clockwise and so the car moves to the right. A spring is attached between the cars. As the cars move apart, the spring is stretched and a force is exerted that pulls the cars back together. This force is found with: 


$$
F=\varsigma\left(X_{r g h t}-X_{l e f t}\right) .
$$

Where $\varsigma$ is the 'elasticity' of the spring and is parameterized from 0 (infinitely elastic, giving $F=0$ ), to 1 (completely rigid so that both cars move as a single unit). It is important to note that $F$ is based on the horizontal distance between the cars as measured in x-units. This will differ from the 'actual' distance due to the changing gradient of the valley slope. Such a difference does not make any substantive difference to the results, but does allow simpler computations. Table 1 lists the parameters of the cable car and Daisyworld models and allows a comparison of the two.

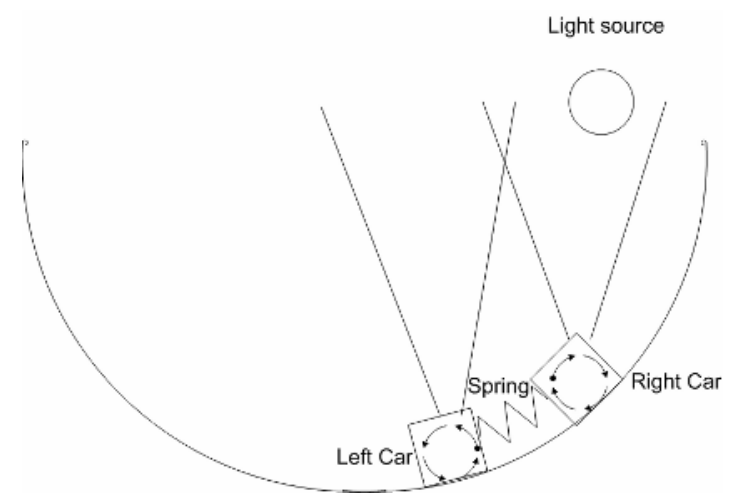

Fig. 3. The left car motor pulls to the left, whilst the right car motor pulls to the right. Depending on the strength of the spring, both cars will move independently or together. The solar panels remain pointing straight up irrespective of the orientation of the cable cars.

Table 1. A comparison of cable car model and Daisyworld parameters

\begin{tabular}{|l|l|}
\hline Cable Car Model & Daisyworld \\
\hline Motor output & Daisy coverage \\
\hline Light location & Luminosity of star \\
\hline Solar panel activation range & Range of viable growth temperatures \\
\hline Left car & Black daisies \\
\hline Right car & White daisies \\
\hline Connecting Spring & Flow of heat from hot to cold \\
\hline
\end{tabular}

\section{Results}

Steady state values over a range of light source positions were found numerically by employing the following algorithm:

1. Calculate the energy produced by the solar panels from the angle of inclination of the light source and therefore the force of rotation of the motors.

2. Calculate the resistance pulling both cars back to the bottom of the valley.

3. Calculate the car's new positions as a sum of the motor output and resisting forces. 
4. Calculate the force exerted by the coupling spring connecting both cars.

5. Move the cars towards a point midway between them in proportion to the coupling spring force.

6. Go back to 1 .

For each time-step, the light source position remained fixed whilst steps 1-6 were iterated until changes in the position of the cable cars were vanishingly small as calculated at double floating point accuracy. In practice 10,000 iterations were sufficient. The width of the activation range was set to $20 \mathrm{x}$-units. The width of the valley was set to $100 \mathrm{x}$-units. The top of the left hand slope was located at $\mathrm{x}=0$, the bottom at $\mathrm{x}=50$ and the top of the right hand slope at $\mathrm{x}=100$. Motor output varied from 0 to a maximum of 1 . $\eta$ was set to 1 in order that $\gamma$ varied linearly from 0 , to a maximum resisting force of 1 when a car was at the top of either slope. The strength of the coupling spring was set to $5 \%, \varsigma=0.05$.

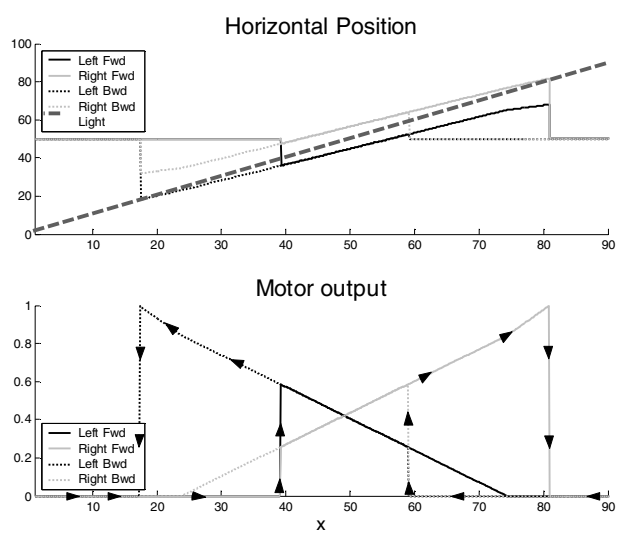

Fig. 4. Solid lines show the car's horizontal position (top plot) and motor output (bottom plot) when the light is moving left to right (forwards). Dashed lines show positions and motor output when the light is moving right to left (backwards). Arrows indicate the hysteresis loops.

As the light moves from left to right, both cars move immediately away from the bottom of the valley. As the light continues to move, the left car slowly moves back down the slope whilst the right car continues to move to the right until its motor output reaches its maximum. As the light moves further, it goes past the centre of the right car's activation range and so solar panel and motor output decreases. This moves the car to the left and so further away from the light source. The right car continues to move back down the slope until it is at rest at the bottom of the valley. As the light reverses direction the same situation occurs but with the right car steadily decreasing in motor output and the left car motor output steadily increasing to its maximum and then abruptly falling to zero. Such behaviour is similar to the growth curves and area of hysteresis observed in Daisyworld simulations in which there is initial rapid 
growth, then steady decline of black daisies whilst the white daisies population slowly increases, peaks and then suddenly collapse.

\subsection{Phototaxis as Homeostasis}

The homeostatic properties of Daisyworld are assessed in terms of the system's ability to regulate global temperatures to within the range in which daisy growth in possible. This is achieved by maximizing the range of luminosity in which daisies are able to grow, and in particular the range in which both daisy types are present. The cable car model instead performs phototaxis. Phototaxis is defined here as the change in the cable car's position in response to changes in the light source's position, with the result that the position of the light source is maintained between the two cars. This is made more apparent if the location of the light source and cars are plotted over time with the light source taking a sinusoidal motion back and forth along the horizontal plane.
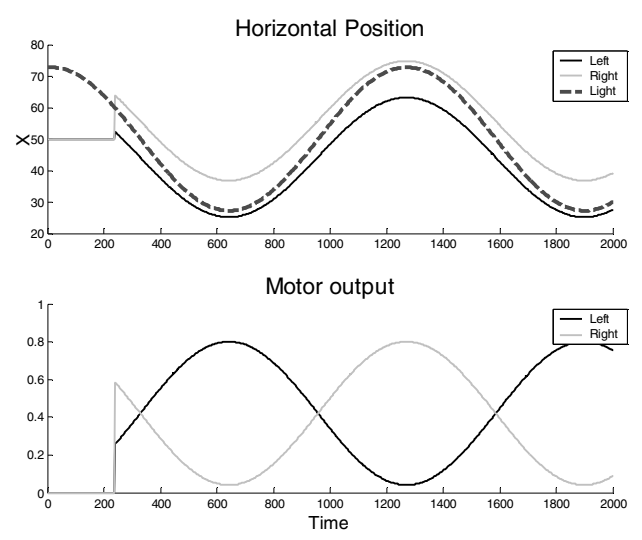

Fig. 5. Time is given in dimensionless units on the horizontal axis on both plots. The top plots shows both cars initially at rest at the bottom of the valley where $x=50$. They are not activated by the light source until time step 210 . The light source moves back and forth along the horizontal plane. The motor outputs and positions of the cars reflect this motion.

Hysteresis is observed when simulations are performed with the light source outside of the activation range of either cable car. Once the cable cars begin to move, they are able to track the light over the entire range of light source positions. If the light source begins within the activation range of either car, or if the right/left hand car is held at its maximum distance from the bottom of the valley, and the light is introduced from the right/left, then there is no period when the cars are inactive. This produces the perhaps counter-intuitive result that decreasing the activation range may not decrease the range of light positions over which phototaxis can be achieved. For example, the hat function could be transformed into a 'spike' function with the result that the light source must be directly overhead in order to produce solar panel output and so motor force. 


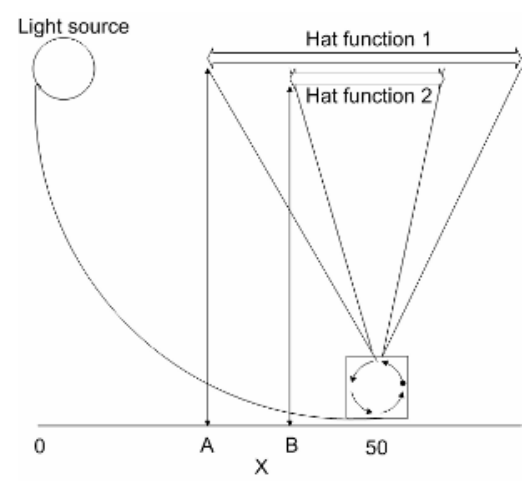

Fig. 6. Two solar panel hat functions for the left hand cable car are plotted. Hat Function 1 is wider than Hat Function 2. The cable car starts at $x=50$ (the bottom of the valley). The light source starts at $\mathrm{x}=0$ (the top of the left hand slope) and moves to the right. Hat Function 1 will be activated at point $\mathrm{A}$, when the light source enters the activation range of the solar panel, whereas Hat Function 2 will not activate until point B. Once the car is activated, if the light source were to reverse direction and move to the left, the limits of phototaxis would be the same for both hat functions.

For a single cable car, the limits of activation are not determined by the width of the hat functions rather their heights and the feedback that the cable cars exert. This feedback is a function of the strength of motor output and the sum of resisting forces pulling the car back towards the bottom of the valley. The limits of phototaxis $X_{\max }$, can be found with:

$$
X_{\text {max }}=\alpha_{\text {max }} \frac{l}{\eta} .
$$

where $\alpha_{\max }$ is the maximum possible motor output. If the left hand car starts at the bottom of a slope of length 50 and has a maximum motor output of 1 with $\eta$ set to 1 , the furthest left it can travel is 50 units. It is not necessary to specify the width of the solar panel activation range in order to determine $X_{\max }$.

\subsection{Decreasing the Range of Phototaxis}

In simulations with two cars, as the light moves back and forth, only one cable car is responsible for the light-following behaviour. This car is referred to as the 'active' car. The other car is pulled up the opposite slope by virtue of the connecting spring. This car is referred to as the 'passive' car. As the activation range increases, the motor output of the passive car increases and so the car pulls more strongly away from the light. This also increases the sum of resisting forces on the active car and so reduces the value of $X_{\max }$. Fig. 7. shows the effects of increasing the width of the activation function to $40 \mathrm{x}$-units and then decreasing it to $4 \mathrm{x}$-units. 

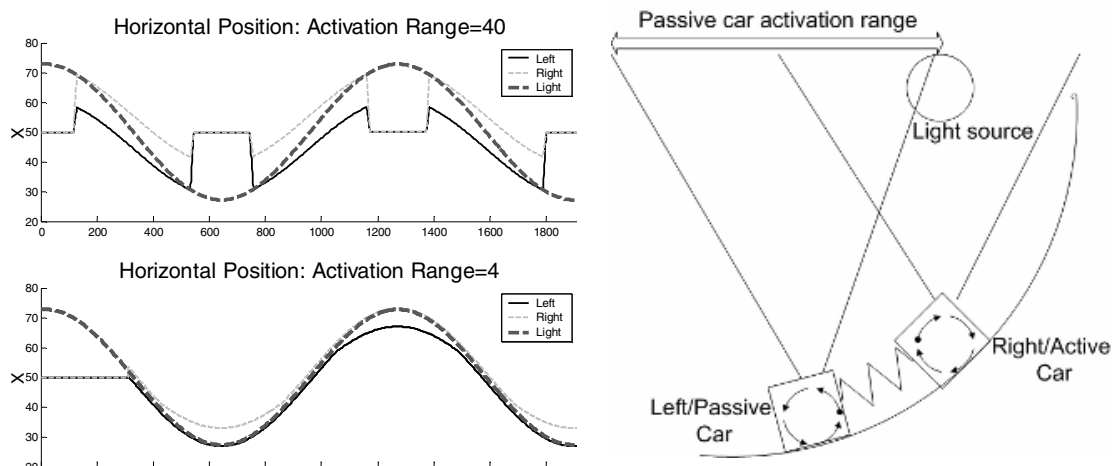

Fig. 7. In the left hand figure, the effects of increasing the activation range to $40 \mathrm{x}$-units are shown in the top plot, the effect of decreasing it to one tenth of this value are shown in the bottom plot. The range of phototaxis is significantly reduced when the activation range is increased to $40 \mathrm{x}$-units. When the activation range is reduced to $4 \mathrm{x}$-units, the distance between the cars and the light source decreases whilst the area of hysteresis increases. In the right hand figure, the effects of increasing the activation range to $40 \mathrm{x}$-units has resulted in the passive car solar panel being activated and so the left hand car pulling away from the light and so increasing the sum resisting forces acting on the active car. This decreases the limits of phototaxis.

\section{Analysis}

The strength of each feedback channel, how hard the motors pull, in part determines the range of external forcing over which the cable car model is able to perform phototaxis. What the channels are pulling against is just as important. Indeed it is the resistance that the cable cars experience as they travel up the valley slope that allows phototaxis to be performed and is also the cause of the observed hysteresis. To explain this will require a moment's anthropomorphising. The roots of hysteresis are found in the different cable car behaviours in response to the light source that is dependent on the direction from which it enters the activation range. For example, if the light source approaches the left car from the right hand side, the car attempts to 'run away' up the slope. It is light-phobic. This is referred to as behaviour A. If however the light source approaches from the left hand side, it is a light-phile. The car runs up the slope to 'meet' the light and then 'follows' it back down the slope. This is referred to as behaviour B. The response of the car is the same under both situations. As the cable car motor is only able to move the car up the slope, the moment the light is detected, the car attempts to 'escape' to the left. During behaviour B, this moves the light across the centre of the activation range, past the mid-point, coming to rest on the right hand side where the output of the motor is balanced by the resisting forces pulling the car back to the bottom of the valley. As the light continues to move to the right, the car follows as the motor output steadily decreases. Due to the coupling spring, the left hand car will continue to move to the right and travel up the opposite 
slope as the right hand car performs its equivalent of behaviour A. Behaviour B is produced by the expenditure of the potential energy that the car stored when the light source first entered the activation range. It is the storing then release of this energy, the balance between $\gamma$ and $\alpha$ as the cars move up and down the energy gradient that produces both behaviours.

As demonstrated in Fig. 7. increasing the essential range can have a dramatic impact on the limits of phototaxis. As the essential range of the cars increase, the sum of resisting forces on the active car increases as the passive car pulls more strongly away from the light source. The active car not only hauls the mass of the passive car up the valley slope, but has to drive against the passive car's motor which attempts to pull the cars in the opposite direction.

\section{Conclusion}

Homeostasis is a core concept for understanding real or artificial life. Rein control is a little known notion of key relevance to homeostasis. Here we have developed this to bring out the new insight that, under some circumstances, increasing the range of tolerable values for essential variables can actually reduce robustness to external perturbation. These are very general observations, but have been discussed in the specific case of the cable car parable.

The cable car model is an intentionally simple system, however the behaviour it exhibits is at times not straightforward. An analysis of the hysteresis observed within this system has focussed on the relationship between the essential range and regulation. It has been shown that decreasing the essential range increases the area of hysteresis whilst the limits of homeostasis remain unchanged. Results have been presented in which increases in the essential range, decreased the range of selfregulation.

In the cable car model, making changes that result in an increase in the area of hysteresis may be desirable if there is an energetic cost associated with moving cable cars. For example, the purpose of the cable cars could be to triangulate a randomly moving light source as part of a targeting system. If the system requires time to active and also to deactivate, then a series of activations followed by deactivations followed by activations would be inefficient. A much better strategy would be to wait until there is a good probability of the target remaining within range for enough time to fully activate the target system. This could be achieved by increasing the area of hysteresis via a decrease in the width of the activation function. The system would be more stable by reducing the range of values it is able to operate within. Once activated, the system would be able to track the target over the same range of values as a system that was configured with a wider activation range.

Hysteresis may be present in a wide variety of processes and mechanisms, for example, the differential operation of a servo moving in opposite directions, or the differential activation threshold of a neuron. In either situation, hysteresis can be regarded as an 'ecological affordance' in that the hysteresis is an implicit element of the agent's body or environment that may be harnessed to allow a desired or evolved set of outputs or behaviours. Analysis of the cable car model has provided insights 
into the relationship between hysteresis and the limits of homeostasis. These insights may be applicable to not only cable car models and Daisyworld but many, if not all, natural or artificial homeostatic systems that operate on the basis of rein control.

\section{References}

1. W. Ross Ashby, Design for a Brain: The Origin of Adaptive Behaviour, Chapman \& Hall Ltd and Science Paperbacks, 1965, 1972.

2. Clynes, Cybernetic Implications of Rein Control in Perceptual and Conceptual Organization. NY Acad. Sci. 156 (1969) pp629-670

3. Harvey I., Homeostasis and Rein Control: From Daisyworld to Active Perception. Proceeding of the Ninth International Conference on the Simulation and Synthesis of Living Systems, ALIFE'9, Pollack K,. Bedau M., Husbands P., Ikegami T. and Watson R.A. (eds) (2004) pp309-314. MIT Press, Cambridge MA

4. Saunders P.T., Koeslag J. and Wessels J.A., Integral Rein Control in Physiology. J. Theo. Biol. 194 (1994) pp 163-173

5. Watson A.J. \& Lovelock J.E., Biological homeostasis of the global environment: the parable of Daisyworld. Tellus 35B (1983) pp284-289 